\title{
Diagnóstico de intolerancia a la lactosa en adultos: rendimiento comparativo de la clínica, test de hidrógeno espirado y test genético
}

\author{
ANTONIO ROLLÁN ${ }^{1}$, CECILIA VIAL ${ }^{2}$, \\ SOLEDAD QUESADA ${ }^{1, \mathrm{a}}$, KARENA ESPINOZA², MARY HATTON ${ }^{2}$, \\ ALONSO PUGA ${ }^{3, b}$, GABRIELA REPETTO ${ }^{2}$
}

\section{Comparative performance of symptoms questionnaire, hydrogen test and genetic test for lactose intolerance}

Background: Genetically programmed adult-type hypolactasia affects 56\% of Chilean population. Ideally, diagnosis should be confirmed. Aim: To compare diagnostic yield of genetic test, hydrogen $\left(\mathrm{H}_{2}\right)$ expiratory test and a validated symptomatic structured survey (SS). Material and Methods: Patients submitted to $\mathrm{H}_{2}$ test answered a historic (anamnestic) and current SS (after the ingestion of $25 \mathrm{~g}$ of lactose). A blood sample was obtained for determination of genetic polymorphisms C/T_13910, C/G_13907 and G/A_22018 by polymerase chain reaction. The gold standard for diagnosis of lactose intolerance (LI) was the agreement of at least two of three tests. Results: Sixty-one participants aged $39 \pm 12$ years (21 males), were studied. Anamnestic SS was diagnostic of LI in all cases (score $\geq 7$ ), while current SS detected LI in 27/61 (46\%). $\mathrm{H}_{2}$ test (an increase $\geq 15$ ppm after ingestion of $25 \mathrm{~g}$ of lactose) showed LI in 31/61 (51\%). The locus C/G_13907 showed no polymorphism and locus G/A_22018 was in complete linkage disequilibrium with C/T_13910. Genotype C/C_13910, associated to hypolactasia, was present in 30/58 (52\%). According to the gold-standard, 32/61 (52.5\%) patients were diagnosed as LI. Sensitivity and specificity were, respectively, 79\% and 69\% for current SS, 93\% and 93\% for $\mathrm{H} 2$ test and $97 \%$ and $93 \%$ for the genetic test. The last two showed a positive likelihood ratio $(L R)>10$ and a negative $L R<0.1$, figures within the range considered clinically useful. Conclusions: Genotype C/C_13910 is responsible for hypolactasia in this population. Anamnestic report of symptoms after milk ingestion and symptoms after lactose ingestion, are not accurate enough. $\mathrm{H}_{2}$ and genetic tests are simple and similarly accurate to diagnose lactose intolerance in adults.

(Rev Med Chile 2012; 140: 1101-1108). naires.

Key words: Genetic testing; lactose intolerance; lactose tolerance test; question-

$\mathrm{M}$ uchos pacientes relacionan sus síntomas digestivos con la ingesta de lácteos. Para diagnosticar intolerancia a la lactosa (IL) debe demostrarse su mala absorción (MAL) en el intestino delgado ${ }^{1}$, la que habitualmente se debe a la disminución de la actividad de lactasa (lactasa-

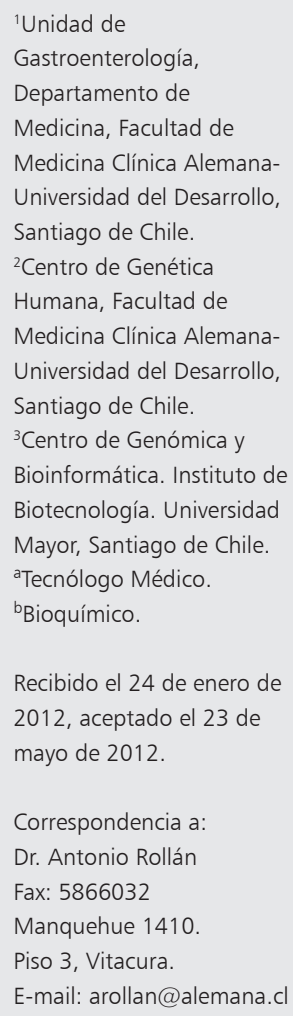

Recibido el 24 de enero de 2012, aceptado el 23 de mayo de 2012.

Correspondencia a: Dr. Antonio Rollán Fax: 5866032 Manquehue 1410. Piso 3, Vitacura. E-mail:arollan@alemana.c

phlorizin hidrolasa) en el ribete en cepillo de los enterocitos (hipolactasia). Este déficit enzimático puede ser adquirido (secundario) o genético (primario), siendo este último mucho más frecuente.

Los niños (como los recién nacidos de cualquier mamífero) expresan alta actividad de lactasa. 
En una proporción variable de la población, luego del destete se produce una reducción genéticamente programada en su síntesis, que determina hipolactasia del adulto ${ }^{2}$. La no persistencia de lactasa (NPL) condiciona que la lactosa alcance el colon donde, al ser metabolizada por las bacterias colónicas, puede generar dolor abdominal, diarrea y meteorismo, aunque la mayoría de los sujetos NPL son asintomáticos. La persistencia de actividad de lactasa (PL) en el adulto es una característica hereditaria dominante, adquirida evolutivamente ${ }^{3}$. Estos conceptos se resumen en la Tabla $1^{4}$.

Recientemente se han identificado los polimorfismos genéticos determinantes del fenotipo PL o NPL. Tres polimorfismos de nucleótido único $(\mathrm{SNP})\left(\mathrm{C} / \mathrm{T} \_13910, \mathrm{G} / \mathrm{A} \_22018\right.$ y C/G_13907) ubicados en regiones intrónicas del gen MCM6, localizado en la región cromosómica 2 q21, regulan la expresión del gen de la lactasa (LCT $)^{5-7}$. La variante C/T_13910 es la principal determinante de PL/NPL en Europa y poblaciones nómades de África. El genotipo C/C_13910 determina fenotipo NPL y C/T o T/T_13910, fenotipo PL ${ }^{6}$. El polimorfismo G/A_22018 se ubica en el intrón 9 del MCM6 y el alelo A se asocia a PL. Este polimorfismo se encuentra en fuerte desequilibrio de ligamiento con el anterior ${ }^{7}$. Otras variantes, como el polimorfismo C/G_13907, parecen ser importantes en algunas poblaciones de África ${ }^{8}$.

La frecuencia poblacional de NPL es variable ${ }^{9,4}$. En el norte de Europa alcanza $2-8 \%{ }^{1}$, aunque pudiera aumentar debido a la inmigracion ${ }^{10}$. En Polonia alcanza a $30 \%{ }^{11}$, en Brasil 67\% (caucásicos y mulatos) ${ }^{12}$ y en afro-americanos en Estados Unidos de Norteamérica hasta $75 \%{ }^{13}$. Poblaciones nativas de América presentan las mayores tasas reportadas ${ }^{9}$. En Chile, un estudio reciente demostró NPL (C/C_13910) en 56\% de población hispana (mestiza) y en $88 \%$ de mapuches ${ }^{14}$. La mayoría de los sujetos NPL son asintomáticos o toleran dosis diarias de lactosa equivalentes a una taza de leche (12 g) y la mayoría de los que reportan intolerancia a lácteos no tienen realmente $\mathrm{MAL}^{9}$.

Los síntomas luego de ingerir leche admiten diversas interpretaciones. La IL (NPL) y los trastornos digestivos funcionales (TDF) comparten el mismo complejo sintomático y pueden coexistir. Comparados con población sana, la IL es más frecuente en sujetos con TDF, mientras que la frecuencia de NPL es la misma. Existe poca correlación entre los síntomas y el resultado de métodos diagnósticos objetivos ${ }^{15}$. Los principales métodos diagnósticos para detectar IL se resumen en la Tabla 1.

El objetivo principal de este estudio fue evaluar prospectivamente el rendimiento diagnóstico de diversos polimorfismos del gen MCM6 en adultos chilenos con síntomas sugerentes de IL, correlacionarlos con MAL y con la presencia de síntomas post ingesta de leche o lactosa.

\section{Material y Métodos}

\section{Pacientes}

Enviados para test de $\mathrm{H}_{2}$ espirado post lactosa por sospecha clínica de IL. Se obtuvo una muestra de sangre y se aplicó un cuestionario de síntomas. Todos participaron voluntariamente y firmaron consentimiento informado específico. El estudio fue aprobado por el Comité de Ética de nuestra institución.

\section{Tabla 1. Definiciones y métodos diagnósticos en intolerancia a lactosa}

$\begin{array}{ll}\begin{array}{l}\text { No persistencia de lactasa (NPL) } \\ \text { (Hipolactasia del adulto) }\end{array} & \begin{array}{l}\text { Disminución o desaparición de la actividad de lactasa en el ribete en cepillo de los } \\ \text { enterocitos. Puede demostrarse determinando la actividad de lactasa en biopsia intes- } \\ \text { tinal (endoscópica) y/o por la detección de polimorfismos específicos en el gen MCM6 } \\ \text { (promotor de la lactasa) }\end{array} \\ \text { Mala absorción de lactosa (MAL) } & \begin{array}{l}\text { Indica que una fracción de la lactosa ingerida alcanza el colon, donde es digerida por } \\ \text { las bacterias endoluminales. Puede demostrarse mediante test de } \mathrm{H}_{2} \text { en aire espirado o } \\ \text { glicemia post carga de lactosa oral }\end{array} \\ \text { Intolerancia a la lactosa (IL) } & \begin{array}{l}\text { Indica la presencia de síntomas como dolor abdominal, diarrea y/o meteorismo aso- } \\ \text { ciados a mala absorción de lactosa. Su diagnóstico requiere evaluación clínica y la } \\ \text { objetivación de MAL y/o NPL }\end{array}\end{array}$




\section{Evaluación clínica y encuesta sintomática estructurada (ESE)}

Se registró el diagnóstico y los síntomas principales. Los pacientes respondieron un cuestionario de síntomas de 5 ítems, tanto anamnéstico, recordando las molestias presentadas al ingerir leche (ESE anamnéstica) como luego de ingerir $25 \mathrm{~g}$ de lactosa (ESE actual). Las molestias se califican en una escala visual análoga (EVA 0-10) y el puntaje total (0-50), se obtiene sumando los 5 ítems. De acuerdo a Casellas y col, utilizando 50 g de lactosa y test espiratorio como gold standard, un puntaje $>6,5$ tiene sensibilidad de $75 \%$ y especificidad de $67 \%$ para $\mathrm{MAL}^{16}$.

\section{Test espiratorio post lactosa}

Luego de $48 \mathrm{~h}$ de dieta baja en fibra, sin lactosa, sin tabaco y ayuno de $12 \mathrm{~h}$ se realizó una determinación de $\mathrm{H}_{2}$ y $\mathrm{CH}_{4}$ basal en aire espirado mediante analizador Quintron (Quintron Instrument Company, Milwaukee, WI, USA), en el Laboratorio de Fisiología Digestiva de Clinica Alemana. Después de ingerir $25 \mathrm{~g}$ de lactosa en $250 \mathrm{ml}$ de agua, se repitieron las determinaciones cada 30 min durante $3 \mathrm{~h}$. Se consideró MAL un aumento $\geq 15$ partes por millón (ppm) de $\mathrm{H}_{2}$ respecto al basal en cualquier determinación ${ }^{17}$. Evaluamos además el rendimiento diagnóstico de la determinación conjunta de $\mathrm{H}_{2} \mathrm{y} \mathrm{CH}_{4}$.

\section{Determinación de polimorfismos gen MCM6}

Se extrajo ADN genómico de sangre periférica mediante ensayo comercial (Axygen CA, USA). Los polimorfismos se estudiaron mediante reacción en cadena de la polimerasa, seguida de digestión por enzimas de restricción y análisis de polimorfismos de longitud de fragmentos (PCR -RFLP) según protocolo descrito previamente ${ }^{2}$. Las determinaciones se realizaron en el Laboratorio de Genética de la Facultad de Medicina Clinica Alemana-Universidad del Desarrollo. El genotipo se determina a partir del tamaño de los fragmentos, analizados en gel de agarosa. Los partidores y enzimas fueron los siguientes:

Polimorfismo C/T-13910: Partidor 1: GCTGGCAATACAGATAAGATAATGGA, Partidor 2: CTGCTTTGGTTGAAGCGAAGAT, Enzima: HinfI (New England Biolabs, MA, USA).

Polimorfismo C/G-13907: Partidor 1: GGATCTCCTTTTGGACTTTCC, Partidor 2: TGGACCTAAACCAATAATGATGAA, Enzima: Hpy8I (Fermentas, MD, USA).
Polimorfismo G/A-22018: Partidor 1: TAAGAACATTTTACACTCTTC, Partidor 2: AGAAAATGGGTTTTCGCCATG, Enzima: HhaI (Fermentas, MD, USA).

\section{Análisis estadístico}

Las variables numéricas fueron descritas mediante promedios y desviación estándar $y$ comparadas mediante test $t$ de Student. Las variables categóricas se describen mediante números y porcentajes y comparadas mediante test de chicuadrado. Las frecuencias genotípicas observadas del locus C/T_13910 se compararon con las esperadas según el equilibrio de Hardy-Weinberg $\left(\mathrm{p}^{2}+2 \mathrm{pq}+\mathrm{q}^{2} ; \mathrm{p}=\right.$ Frecuencia alelo $\mathrm{T} ; \mathrm{q}=$ Frecuencia alelo $\mathrm{C})$. Utilizando como gold standard la concordancia de dos de tres test utilizados, calculamos sensibilidad, especificidad y razones de verosimilitud (likelihood ratio (LR)) positivas y negativas para cada uno de los test diagnósticos. Se consideró significativo $\mathrm{p}<0,05$.

\section{Resultados}

Se incluyeron 61 pacientes, cuyos datos demográficos, sintomatología y patología asociada se resumen en la Tabla 2.

Cincuenta y ocho pacientes (95\%) ingerían habitualmente leche y/o derivados, aunque 52 (85\%) referían molestias digestivas luego de consumirla y $11(18 \%)$ sólo consumían leche sin lactosa. Diecisiete pacientes (28\%) reportaron familiares directos con IL.

\section{Encuesta sintomática estructurada (ESE)}

La ESE anamnéstica y actual se resumen en la Figuras 1 y 2. El meteorismo y el borborigmo fueron los síntomas más relevantes, mientras vómitos o diarrea fueron excepcionales en la ESE actual. El puntaje total promedio en la ESE anamnéstica fue significativamente mayor que en la ESE actual $(19,4$ vs 9,$5 ; \mathrm{p}<0,05)$ y todos los pacientes (menos uno que no respondió) cumplían el criterio para diagnosticar IL (puntaje $\geq 7$ ), mientras sólo 27 pacientes $(46 \%)$ tuvieron puntaje $\geq 7$ en la ESE actual $(\mathrm{p}<0,05)$ y 11 pacientes $(19 \%)$ no refirieron molestia alguna. La ESE anamnéstica tuvo una sensibilidad de $93,1 \%$, pero una especificidad de $3,5 \%$. El rendimiento diagnóstico de la ESE actual se resume en la Tabla 4. 
Tabla 2. Demografía, sintomatología y patología asociada ( $n=61)$

\begin{tabular}{|ll|}
\hline Edad, promedio \pm DE (rango) & $39 \pm 12$ (20-65 años) \\
\hline Sexo, H/M & $21 / 40$ \\
Sintomatología:* & \\
$\quad$ Distensión. Meteorismo. Flatulencia, n (\%) & $46(79 \%)$ \\
$\quad$ Diarrea intermitente, n (\%) & $26(45 \%)$ \\
Dolor abdominal episódico, n (\%) & $27(47 \%)$ \\
Otros, n (\%) & $6(10 \%)$ \\
(dispepsia, náuseas, regurgitación) & \\
Patología asociada: & $8(14 \%)$ \\
$\quad$ Reflujo gastro-esofágico, n (\%) & $3(5 \%)$ \\
$\quad$ Síndrome Intestino Irritable, n (\%) & $7(12 \%)$ \\
\hline Otras**, n (\%) & \\
\hline
\end{tabular}

*Más de un síntoma por paciente. **Constipación (1), Glomerulonefritis (1), Disautonomia (1), Esplenomegalia congénita (1). Intolerancia a glucosa (1). Alergia alimentaria (1), Espondilitis anquilosante (1).

Tabla 3. Frecuencia genotípica locus MCM6 C/T_13910 ( $\mathbf{n}=\mathbf{5 8})$

\begin{tabular}{|ccc|}
\hline Frecuencia genotípica & Observada $\mathbf{n}(\%)$ & Esperada $\mathbf{n}(\%) *$ \\
C/C_13910 & $30(51,7 \%)$ & $25(42,9 \%)$ \\
C/T_13910 & $16(27,6 \%)$ & $26(45,2 \%)$ \\
\hline T/T_13910 & $12(20,7 \%)$ & $7(11,9 \%)$ \\
\hline
\end{tabular}

$\mathrm{p}=$ NS (Observada vs Esperada). *Según Ley de Hardy-Weinberg (ver Métodos).

\section{Test de $\mathrm{H}_{2}$ espirado post lactosa}

El test de $\mathrm{H}_{2}$ espirado post $25 \mathrm{~g}$ de lactosa demostró MAL en 31 pacientes $(51 \%)$. La suma de $\mathrm{H}_{2}$ y $\mathrm{CH}_{4} \geq 16$ ppm o de $\mathrm{H}_{2}$ y $2 \mathrm{x} \mathrm{CH}_{4} \geq 17 \mathrm{ppm}^{18}$ no mejoró el rendimiento diagnóstico en comparación con la determinación exclusiva de $\mathrm{H}_{2}$, (datos no mostrados). El rendimiento diagnóstico se muestra en la Tabla 4.

\section{Polimorfismos gen MCM 6}

Evaluamos 58 pacientes (en 3 pacientes no fue posible realizar la determinación). La Figura 3 muestra un gel representativo de los diferentes genotipos. El locus C/G_13907 no mostró polimorfismo en esta población. El locus A/G_22018 mostró un completo desequilibrio de ligamiento con el locus C/T_13910. Los análisis posteriores se efectuaron considerando sólo el locus C/T_13910. Se demostró genotipo C/C_13910, en $30 / 58$ pacientes $(52 \%)$. La Tabla 3 muestra las frecuencias genotípicas

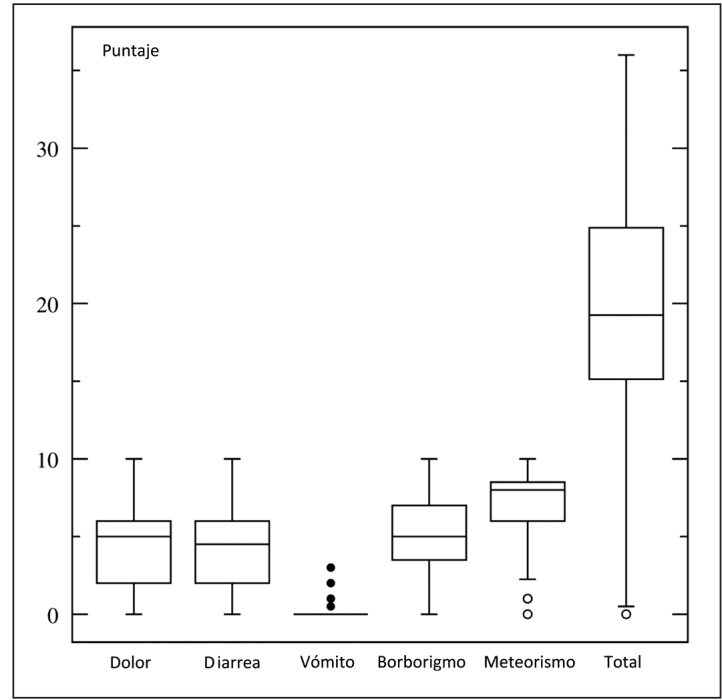

Figura 1. Box-plot encuesta sintomática anamnéstica (Histórica). La caja muestra la mediana (línea central) y los percentiles 25 y 75 (primer y tercer cuartil respectivamente). Las extensiones verticales ("whiskers") comprenden hasta 1,5 veces el rango intercuartil. Los círculos muestran datos fuera de este rango ("outliers").

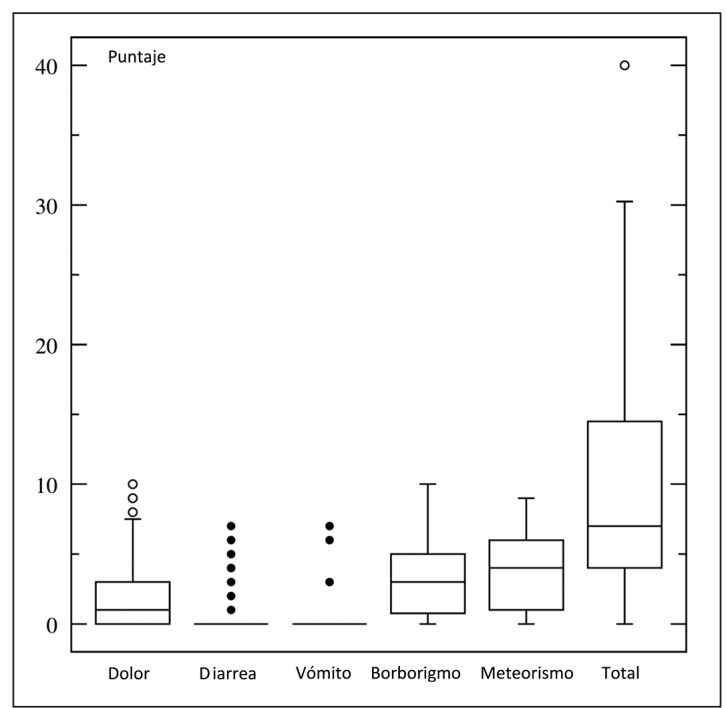

Figura 2. Box-plot encuesta sintomática actual (post ingesta de $25 \mathrm{~g}$ de lactosa). La caja muestra la mediana (línea central) y los percentiles 25 y 75 (primer y tercer cuartil respectivamente). Las extensiones verticales ("whiskers") comprenden hasta 1,5 veces el rango intercuartil. Los círculos muestran datos fuera de este rango ("outliers"). 
Diagnóstico intolerancia a lactosa en adultos - A. Rollán et al

Tabla 4. Rendimiento de los métodos diagnósticos para Intolerancia a lactosa*

\begin{tabular}{|lcccccc|}
\hline Test Dx & $\begin{array}{c}\text { Sensibil. \% } \\
(\mathbf{I C ~ 9 5 \% )}\end{array}$ & $\begin{array}{c}\text { Especific. \% } \\
\text { (IC 95\%) }\end{array}$ & $\begin{array}{c}\text { VPP \% } \\
\text { (IC 95\%) }\end{array}$ & $\begin{array}{c}\text { VPN \% } \\
\text { (IC 95\%) }\end{array}$ & $\begin{array}{c}\text { LR (+) } \\
\text { (IC 95\%) }\end{array}$ & $\begin{array}{c}\text { LR (-) } \\
\text { (IC 95\%) }\end{array}$ \\
Síntomas post lactosa & 79 & 69 & 72 & 77 & 2,6 & 0,3 \\
(ESE $\geq 7)$ & $(63-96)$ & $(50-88)$ & $(55-89)$ & $(59-95)$ & $(1,4-4,5)$ & $(0,1-0,6)$ \\
Test espiratorio & 93 & 93 & 93 & 93 & 13,5 & 0,07 \\
$\left(\mathrm{H}_{2} \geq 15\right.$ ppm) & $(82-100)$ & $(82-100)$ & $(82-100)$ & $(82-100)$ & $(3,5-51,6)$ & $(0-0,28)$ \\
Test genético & 97 & 93 & 93 & 96 & 14 & 0,04 \\
(C/C_13910) & $(88-100)$ & $(82-100)$ & $(82-100)$ & $(88-100)$ & $(3,7-53,4)$ & $(0-0,25)$ \\
\hline
\end{tabular}

${ }^{*}$ Gold-standard: concordancia de $2 / 3$ test. VPP $=$ Valor Predictivo $(+)$; VPN $=$ Valor Predictivo $(-)$. LR $(+)=$ Razón de verosimilitud (+); LR (-) = Razón de verosimilitud (-).

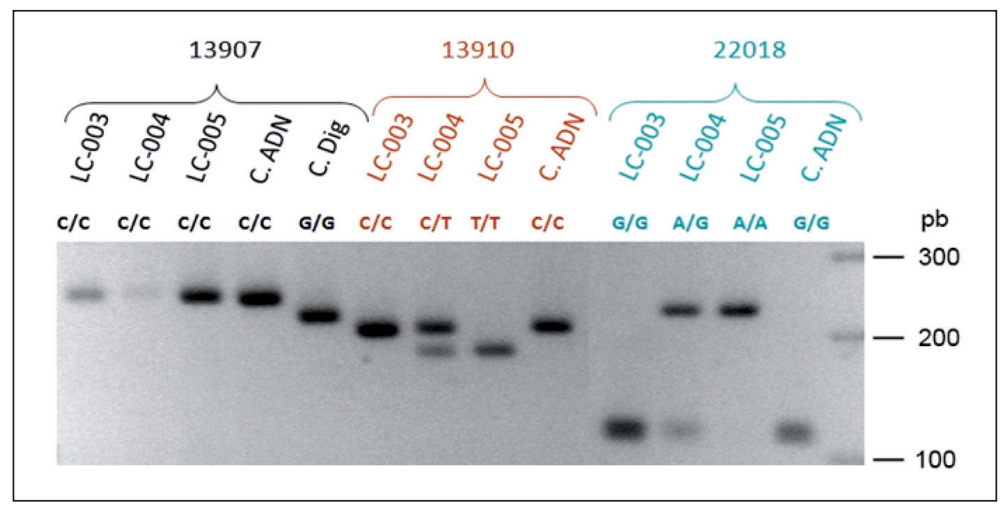

Figura 3. Polimorfismos gen MCM6 (PCR-RFLP). LC-003, LC-004 y LC-005: Identificación de tres pacientes distintos. C. ADN, C.Dig: Controles internos. observadas y las esperadas de acuerdo a la ley de Hardy-Weinberg. Las frecuencias de C/C (51,7\%), $\mathrm{C} / \mathrm{T}(27,6 \%)$ y $\mathrm{T} / \mathrm{T}(20,7 \%)$ se encuentran en equilibrio de Hardy-Weinberg en esta población $\left(\chi^{2}=4,15 ; \mathrm{p}=0,12\right)$. El rendimiento diagnóstico del test genético se muestra en la Tabla 4 .

\section{Rendimiento Diagnóstico}

El test espiratorio y el test genético coincidieron en 52/58 pacientes (90\%). En 4/6 pacientes discordantes el diagnóstico final (determinado por el gold standard) coincidió con el test genético y en uno de los 2 restantes el test espiratorio mostró elevación significativa de $\mathrm{CH}_{4}$, de modo que eventualmente 5 de los 6 discordantes habrían sido correctamente diagnosticados por el test genético.

De acuerdo al gold standard, 32 pacientes $(52,5 \%)$ fueron diagnosticados como IL. En los 3 pacientes en que no se contó con el test genético los otros dos tests fueron coincidentes ( 2 tolerantes y 1 intolerante), de modo que fueron incluidos en el análisis.
La Tabla 4 muestra el rendimiento diagnóstico global. Tanto el test espiratorio como el test genético muestran un rendimiento muy elevado $y$ comparable. El rendimiento de la ESE actual fue menor y los LR no alcanzan los niveles requeridos para recomendar su uso clínico, aunque no es posible demostrar diferencias significativas entre los tres tests (ver IC 95\% en Tabla 4), probablemente por el reducido tamaño muestral.

\section{Discusión}

La frecuencia de IL en esta muestra de pacientes sintomáticos $(52,5 \%)$ resultó muy similar al $56 \%$ esperado para población hispánica (mestiza) chilena ${ }^{14}$. Si bien no puede descartarse que la población de origen tenga una menor prevalencia de NPL, el que los genotipos se encuentren en equilibrio de Hardy-Weinberg sugiere que la muestra es similar a la población original. La historia de síntomas post ingesta de leche tiene 
escasa utilidad para seleccionar pacientes con NPL y MAL, como ha sido repetidamente reportado en la literatura ${ }^{15,18,19}$.

En la práctica clínica habitual los síntomas asociados a la ingesta de leche pueden atribuirse a NPL, a TDF o a una mezcla de ambos. Lo recomendable pareciera ser evaluar la respuesta a la supresión de lactosa: si los síntomas desaparecen no es clara la necesidad de una mayor precisión diagnóstica, dado el bajo riesgo de una patología orgánica significativa. Sin embargo, la supresión de lactosa es difícil de sostener en sujetos con alta apetencia por la leche y la respuesta clínica es a veces incompleta o inconsistente. La compleja relación entre síntomas y dieta en pacientes con TDF puede originar una innecesaria supresión de lácteos en una alta proporción de la población. Un reciente consenso del National Institute of Health (NIH, USA) advierte sobre el riesgo de un inadecuado consumo de calcio y vitamina $\mathrm{D}$ en estos pacientes ${ }^{9}$. Esta advertencia parece aun más relevante en países más pobres y con alta frecuencia de NPL, sugiriendo la conveniencia de confirmar IL, al menos en los casos en que la respuesta clínica no sea bien clara, lo que implica la necesidad de validar métodos diagnósticos objetivos.

Como ya fue señalado (Tabla 1), los métodos disponibles evalúan aspectos diferentes del problema, lo que impide contar con un gold standard objetivo. Al igual que en otros estudios similares, escogimos la concordancia entre los métodos para calificar el estado final de nuestros pacientes, lo que constituye una aproximación aceptada para evaluar el rendimiento de diversos métodos diagnósticos cuando no existe un gold standard aceptado $^{21}$.

La Encuesta Sintomática Estructurada constituye un instrumento atractivo por su simplicidad, bajo costo y facilidad de aplicación. Casellas ${ }^{16}$, utilizando $50 \mathrm{~g}$ de lactosa y test espiratorio como gold standard, demostró sensibilidad de $75 \%$ y especificidad de $67 \%$. Si bien nosotros utilizamos sólo $25 \mathrm{~g}$ de lactosa obtuvimos resultados muy similares (sensibilidad 79\% y especificidad 69\%). Aunque el rendimiento diagnóstico fue muy superior a la ESE anamnéstica, los LR de la ESE post lactosa actual (Tabla 4) no alcanzan los niveles aceptados para recomendar su uso clínico $(\operatorname{LR}(+)>10 \text { y } \operatorname{LR}(-)<0,1)^{21}$.

El test espiratorio y el test genético tuvieron un rendimiento diagnóstico elevado y similar en este estudio. Ambos métodos muestran valores de LR (+) y (-) en rangos considerados clínicamente útiles. Si bien el test espiratorio no está bien estandarizado y existen importante diferencias en su aplicación, incluyendo la dosis de lactosa, el considerar o no el $\mathrm{CH}_{4}$, los puntos de corte a utilizar y además múltiples aspectos, tales como las característica de la flora intestinal, la dieta previa y el tabaco, pueden influir en su rendimiento, diversos estudios coinciden en reportar un excelente rendimiento diagnóstico, por lo que se ha constituido en el método preferido en la mayor parte de los centros ${ }^{18}$. Un reciente consenso ha sugerido recomendaciones para estandarizar su aplicación, incluyendo considerar sólo los niveles de $\mathrm{H}_{2}$ y no los de $\mathrm{CH}_{4}$, lo que coincide con nuestros resultados ${ }^{17}$. Las principales ventajas del test espiratorio son su naturaleza no invasiva y la ausencia de riesgos, mientras su limitación más importante es la necesidad de restricciones dietéticas previas y la duración del test (hasta 4 h) ${ }^{17}$.

Coincidiendo con un estudio previo en Chile ${ }^{14}$, nuestros resultados confirman que la mutación europea (C/T_13910) es la principal determinante de PL/NPL en nuestra población. La determinación genética presenta ventajas de simplicidad (sólo requiere una muestra de $\mathrm{ADN}$, sin restricción dietética alguna) y de interpretación (el resultado es claramente positivo o negativo) que la hacen atractiva para su uso clínico. Potenciales desventajas son, sin embargo, que el genotipo C/C_13910 no necesariamente implica MAL, ya que el "switch off" del gen pudiera ocurrir a edades variables y la posibilidad de que existan otros genotipos asociados al fenotipo MAL. La excelente correlación que observamos entre ambos métodos (genético y respiratorio) y el óptimo rendimiento del test en esta muestra sugieren que estas objeciones son teóricas, al menos en el adulto. En niños con genotipo C/C_13910, un estudio reciente en India mostró que la supresión de la lactasa se inicia entre los 3 y 5 años y que a los 8 años de edad, todos los niños mostraban una actividad muy reducida ${ }^{22}$. Otro estudio en niños africanos y finlandeses sugiere que la disminución de la actividad enzimática pudiera ser más precoz en niños africanos ${ }^{23}$. Estos datos deben ser corroborados en otras poblaciones y en nuestro centro estamos iniciando un protocolo al respecto. La principal desventaja del método genético es su escasa disponibilidad, la necesidad de laboratorios especializados y su alto costo. La 
posibilidad de obtener ADN de muestra de saliva o de un frotis de mucosa oral permitiría mejorar aún más su aceptación y eventualmente promover una eventual disminución de costos.

En resumen, este estudio de pacientes con síntomas sugerentes de IL confirma que aun en poblaciones con alta frecuencia de hipolactasia el antecedente anamnéstico tiene escaso valor diagnóstico y que la cuantificación de síntomas inmediatamente después de ingerir lactosa tampoco alcanza un rendimiento diagnóstico suficiente. Confirmamos que la alta frecuencia de NPL en nuestra población se asocia al polimorfismo C/T_13910 del gen MCM6 y demostramos un alto rendimiento diagnóstico del test genético, comparable o superior al test espiratorio y con claras ventajas de administración e interpretación.

\section{Referencias}

1. Jellema P, Schellevis FG, van der Windt DAWM, Kneepkens CMF, van der Horst HE. Lactose malabsorption and intolerance: a systematic review on the diagnostic value of gastrointestinal symptoms and self-reported milk intolerance. QJM 2010; 103 (8): 555-72.

2. Swallow DM. Genetics of lactase persistence and lactose intolerance. Annu Rev Genet 2003; 37: 197-219.

3. Sahi T. The inheritance of selective adult-type lactose malabsorption. Scand J Gastroenterol Suppl 1974; 30: 1-73.

4. Wilt TJ, Shaukat A, Shamliyan T, Taylor BC, MacDonald R, Tacklind J, Rutks I, Schwarzenberg SJ, Kane RL, Levitt M. Lactose intolerance and health. Evid Rep Technol Assess (Full Rep) 2010; (192): 1-410.

5. Wang Y, Harvey CB, Pratt WS, Sams VR, Sarner M, Rossi M, et al. The lactase persistence/non-persistence polymorphism is controlled by a cis-acting element. Hum Mol Genet 1995; 4 (4): 657-62.

6. Enattah N-S, Kuokkanen M, Forsblom C, Natah S, Oksanen A, Jarvela I, et al. Correlation of intestinal disaccharidase activities with the $\mathrm{C} / \mathrm{T}-13910$ variant and age. World J. Gastroenterol 2007; 13 (25): 3508-12.

7. Enattah NS, Sahi T, Savilahti E, Terwilliger J, Peltonen L, Järvelä I. Identification of a variant associated with adult-type hypolactasia. Nat Genet 2002; 30 (2): 233-7.

8. Tishkoff SA, Reed FA, Ranciaro A, Voight B, Babbitt C, Silverman JS, et al. Convergent adaptation of human lactase persistence in Africa and Europe. Nat Genet 2007; 39 (1): 31-40.

9. Brannon PM, Carpenter TO, Fernández JR, Gilsanz V, Gould JB, Hall KE, et al. NIH Consensus Develop- ment Conference Statement: Lactose Intolerance and Health. NIH Consens State Sci Statements. 2010; 27 (2). Disponible en: http://www.ncbi.nlm.nih.gov/pubmed/20186234. Accedido 19 de Julio de 2011.

10. Almon R, Engfeldt P, Tysk C, Sjöström M, Nilsson TK. Prevalence and trends in adult-type hypolactasia in different age cohorts in Central Sweden diagnosed by genotyping for the adult-type hypolactasia-linked LCT -13910C > T mutation. Scand J Gastroenterol 2007; 42 (2): 165-70.

11. Mądry E, Lisowska A, Kwiecień J, Marciniak R, KorzonBurakowska A, Drzymała-Czyż S, et al. Adult-type hypolactasia and lactose malabsorption in Poland. Acta Biochim Pol 2010; 57 (4): 585-88.

12. Mattar R, Mazo DF de C. Lactose intolerance: changing paradigms due to molecular biology. Rev Assoc Med Bras 2010; 56 (2): 230-36.

13. Byers KG, Savaiano DA. The myth of increased lactose intolerance in African-Americans. J Am Coll Nutr 2005; 24 (6 Suppl): 569S-73S.

14. Morales E, Azócar L, Maul X, Pérez C, Chianale J, Miquel JF. The European lactase persistence genotype determines the lactase persistence state and correlates with gastrointestinal symptoms in the Hispanic and Amerindian Chilean population: a case-control and population-based study. BMJ Open 2011; 1 (1): e000125.

15. Casellas F, Aparici A, Casaus M, Rodríguez P, Malagelada JR. Subjective perception of lactose intolerance does not always indicate lactose malabsorption. Clin Gastroenterol Hepatol 2010; 8 (7): 581-6.

16. Casellas F, Varela E, Aparici A, Casaus M, Rodríguez P. Development, validation, and applicability of a symptoms questionnaire for lactose malabsorption screening. Dig Dis Sci 2009; 54 (5): 1059-65.

17. Gasbarrini A, Corazza GR, Gasbarrini G, Montalto M, Di Stefano M, Basilisco G, et al. Methodology and indications of H2-breath testing in gastrointestinal diseases: the Rome Consensus Conference. Aliment Pharmacol Ther 2009; 29 Suppl 1:1-49.

18. Hovde $\varnothing$, Farup PG. A comparison of diagnostic tests for lactose malabsorption-which one is the best? BMC Gastroenterol 2009; 9: 82.

19. Vernia P, Marinaro V, Argnani F, Di Camillo M, Caprilli R. Self-reported milk intolerance in irritable bowel syndrome: what should we believe? Clin Nutr 2004; 23 (5): 996-1000.

20. Rollán A, Giancaspero R, Arrese M, Figueroa C, Vollrath $\mathrm{V}$, Schultz M, et al. Accuracy of invasive and noninvasive tests to diagnose Helicobacter pylori infection after antibiotic treatment. Am J Gastroenterol 1997; 92 (8): 1268-74. 
21. Stengel D, Bauwens K, Sehouli J, Ekkernkamp A, Porzsolt F. A likelihood ratio approach to metaanalysis of diagnostic studies. J Med Screen 2003; 10 (1): 47-51.

22. Kuchay RA, Thapa BR, Mahmood A, Mahmood S. Effect of C/T -13910 cis-acting regulatory variant on expression and activity of lactase in Indian children and its implication for early genetic screening of adult-type hypolactasia. Clin Chim Acta 2011; 412 (21-22): 192430.

23. Rasinperä H, Savilahti E, Enattah NS, Kuokkanen M, Tötterman N, Lindahl H, et al. A genetic test which can be used to diagnose adult-type hypolactasia in children. Gut 2004; 53 (11): 1571-6. 